\title{
HOLOMORPHIC INJECTIVITY AND THE HOPF MAP
}

\author{
SCOTT NOLLET AND FREDERICO XAVIER
}

Warmly dedicated to Joana

\begin{abstract}
We give sharp conditions on a local biholomorphism $F: X \rightarrow \mathbb{C}^{n}$ which ensure global injectivity. For $n \geq 2$, such a map is injective if for each complex line $l \subset \mathbb{C}^{n}$, the pre-image $F^{-1}(l)$ embeds holomorphically as a connected domain into $\mathbb{C P}^{1}$, the embedding being unique up to Möbius transformation. In particular, $F$ is injective if the pre-image of every complex line is connected and conformal to $\mathbb{C}$. The proof uses the topological fact that the natural map $\mathbb{R} \mathbb{P}^{2 n-1} \rightarrow \mathbb{C} \mathbb{P}^{n-1}$ associated to the Hopf map admits no continuous sections and the classical Bieberbach-Gronwall estimates from complex analysis.
\end{abstract}

\section{INTRODUCTION}

The study of univalence (injectivity) of holomorphic functions of one variable is a classical topic in complex analysis. One of the high points in the subject was the celebrated solution of the Bieberbach Conjecture by de Branges in 1984 [2, 5, 7]. As part of the effort to understand the conjecture, several authors introduced various univalence criteria for locally univalent holomorphic functions defined on the open unit disc in $\mathbb{C}$ [17]. In contrast, fewer injectivity criteria are known for local biholomorphisms in higher dimensions [5, chapter V]. In the simplest case when $F: \mathbb{C}^{n} \rightarrow \mathbb{C}^{n}$ is a polynomial local biholomorphism, it is not even known whether $F$ is a priori injective (hence bijective [1, I, Theorem. 2.1]) without further hypotheses: this is the jacobian conjecture (see [1, 4] for general references), which remains open after more than 60 years.

In this paper we draw a connection between global injectivity of a local biholomorphism $F$ and connectedness of certain pre-images of $F$. The naive observation that $F$ is injective if and only if the pre-image of each point is connected leads one naturally to ask whether there might be a similar criterion based on connectedness of pre-images of positive dimensional submanifolds. For example, it's easy to see that a local diffeomorphism $F: X \rightarrow \mathbb{R}^{n}$ is injective if for each real line $l$, the pre-image $F^{-1}(l)$ is connected [15, Example 3.3]. For a local biholomorphism $F: X \rightarrow \mathbb{C}^{n}$ and complex lines $l \subset \mathbb{C}^{n}$, it turns out that connectedness of the pre-images is not enough (Example 2.5), one also needs information about the conformal type of the pre-images $F^{-1}(l)$ (Corollarie 1.2 and 1.3).

To state our injectivity criterion, we need the concept of a rigid domain of $\mathbb{C P}^{1}$ : for us, these are the connected open sets $i: U \hookrightarrow \mathbb{C P}^{1}$ such that any holomorphic embedding $f: U \hookrightarrow \mathbb{C P}^{1}$ differs from $i$ by an automorphism $M$ of $\mathbb{C P}^{1}$, that is $f=M \circ i$. A rigid domain $U \subset \mathbb{C P}^{1}$ is necessarily dense (otherwise apply an automorphism of $\mathbb{C P}^{1}$ that takes $U$ into the unit disc $D \subset \mathbb{C}$. By the Riemann mapping theorem, there are many holomorphic

Work of the second author was partially supported by NSF grant DMS02-03637. 
embeddings $F: D \hookrightarrow \mathbb{C} \subset \mathbb{C P}^{1}$ which are not the restriction of a Möbius transformation. By unicity of continuation, the restriction $\left.F\right|_{U}$ is not either). Typical examples include $\mathbb{C} \subset \mathbb{C P}^{1}$, the complement of $\mathbb{C}$ by finitely many points (a connected rational curve, in the sense of algebraic geometry) or, more generally, the complement in the Riemann sphere of a removable closed set (a closed subset $E \subset \mathbb{C P}^{1}$ is removable if for each open set $U \subset \mathbb{C P}^{1}$, the bounded holomorphic functions $f: U-E \rightarrow \mathbb{C}$ extend holomorphically to $U$ ).

Theorem 1.1. Let $X$ be a connected complex manifold of dimension $n \geq 2, F: X \rightarrow \mathbb{C}^{n} a$ local biholomorphism. Fix $q \in F(X)$ and suppose that $F^{-1}(l)$ is conformal to a rigid domain $D_{l} \subset \mathbb{C P}^{1}$ for every complex line $l$ passing through $q$. Then $q$ is assumed exactly once by $F$.

The statement is sharp in all respects, starting with the obvious condition $n \geq 2$. Its conclusion may fail if even a pre-image of a single line is disconnected (Example 2.4) or if the pre-images are conformal to punctured compact Riemann surfaces of positive genera (Example 2.5). The rigidity condition also cannot be removed (Example 2.7). The proof is a blend of analytic and topological ideas: if $F^{-1}(q)$ contains two points, we can construct a continuous section to the natural map $\pi: \mathbb{R P}^{2 n-1} \rightarrow \mathbb{C P}^{n-1}$ associated to the Hopf map, an impossibility. The continuity of this section follows from the classical Bieberbach-Gronwall estimates for univalent functions on the unit disc. Globalizing Theorem 1.1 yields:

Corollary 1.2. Let $F: X \rightarrow \mathbb{C}^{n}$ be a local biholomorphism with $n \geq 2$. If each non-empty pre-image of a complex line is connected and conformal to $\mathbb{C}$, then $F$ is injective.

Applying Serre's GAGA principle [18] gives an algebro-geometric variant:

Corollary 1.3. Let $F: X \rightarrow \mathbb{A}_{\mathbb{C}}^{n}$ be an étale morphism of schemes with $n \geq 2$. Then the following are equivalent.

(1) $F$ is injective.

(2) For each line $l \subset \mathbb{C}^{n}$ meeting $F(X)$, the pre-image $F^{-1}(l)$ is connected and rational.

The forward direction is clear because the pre-image of a line $l$ is identified with a Zariski open subset of $l$; the converse is immediate from Theorem 1.1 above. Example 2.5] shows that rationality is needed in condition (2) above.

Finally, applying generic smoothness gives a projective version:

Corollary 1.4. Let $F: Z \rightarrow \mathbb{P}_{\mathbb{C}}^{n}$ be a generically finite morphism of schemes with $n \geq 2$ and $Z$ smooth. Then the following are equivalent.

(1) F is birational.

(2) There is an open set $U \subset \mathbb{P}^{n}$ such that for each line $l \subset \mathbb{P}^{n}$, the pre-image $F^{-1}(l \cap U)$ is either empty or irreducible and rational.

Proof. Since $\mathbb{C}$ has characteristic zero, generic smoothness [8, III, Cor. 10.7] gives an open set $V \subset \mathbb{P}^{n}$ for which the restricted map $F: F^{-1}(V) \rightarrow V$ is smooth. Generic finiteness of $F$ implies that (a) the set $V$ is non-empty (hence dense) and (b) the relative dimension is zero so that $F: F^{-1}(V) \rightarrow V$ is étale. Further restriction to a standard open affine subset $\mathbb{A}^{n} \cong U_{i} \subset \mathbb{P}^{n}$ gives an étale morphism $F^{-1}\left(V \cap \mathbb{A}^{n}\right) \rightarrow V \cap \mathbb{A}^{n} \hookrightarrow \mathbb{A}^{n}$.

$\Rightarrow$ : If $F$ is birational, then $\operatorname{deg} F=1$ and we may take $U=V \cap \mathbb{A}^{n}$.

$\Leftarrow$ : The second condition continues to hold if we replace $U$ with $U \cap V \cap \mathbb{A}^{n}$. Composing 
with the inclusion into $\mathbb{A}^{n}$, Corollary 1.3 implies that $\left.F\right|_{F^{-1}\left(U \cap V \cap \mathbb{A}^{n}\right)}$ is injective, hence $F$ is birational.

In section two we prove Theorem 1.1. modulo a delicate continuity proof. We also give examples which show that the hypotheses cannot easily be removed. Section three is devoted to the continuity proof. We direct the interested reader to [12, 15, 16, 19, 20] and the references therein for further results on global injectivity in the differentiable context.

Acknowledgements. In an earlier version of this paper, the conclusion of Theorem 1.1 was that the point $q$ can be assumed at most twice by $F$ and there was an example intended to show that $q$ could have two points in its pre-image. János Kollár generously pointed out how the example failed and suggested in broad terms how our method could be strengthened to yield the optimal result. He also indicated that Corollary [1.3 can be approached using ideas from Mori theory [11, 14.

We would also like to thank Francis Connolly, Richard Hind, and Sean Keel for useful conversations.

\section{MAIN IDEAS AND EXAMPLES}

In this section we prove Theorem 1.1 except for a lengthy technical matter which is delayed to section three. We also give examples to show that the hypotheses cannot be weakened.

Recall that the Hopf map $S^{2 n-1} \rightarrow \mathbb{C P}^{n-1}$ sends a unit vector $u \in \mathbb{C}^{n}$ to the complex one-dimensional subspace containing it. Clearly this induces a map $\pi: \mathbb{R P}^{2 n-1} \rightarrow \mathbb{C P}^{n-1}$ between real and complex projective spaces.

Fact 2.1. Neither $\pi$ nor the Hopf map admits a continuous section for $n \geq 2$.

For instance, the composite map in cohomology

$$
H^{2}\left(\mathbb{C P}^{n-1}\right) \rightarrow H^{2}\left(\mathbb{R P}^{2 n-1}\right) \rightarrow H^{2}\left(\mathbb{C P}^{n-1}\right)
$$

induced by a continuous section of $\pi$ must be the identity, but this is impossible since $H^{2}\left(\mathbb{C P}^{n-1}\right) \neq 0$ and $H^{2}\left(\mathbb{R P}^{2 n-1}\right)=0$.

This topological fact can sometimes be used to prove injectivity of local biholomorphisms. The following example illustrates the idea.

Example 2.2. Let $F: \mathbb{C}^{n} \rightarrow \mathbb{C}^{n}$ be a local bihilomorphism with $n \geq 2$ such that the pre-image of every complex line is connected and simply connected. Then $F$ is injective.

Proof. If $F$ is not injective, we may suppose that $F(p)=F(q)=0$ with $p \neq q$, hence $F^{-1}(l)$ contains both $p$ and $q$ for all one-dimensional complex subspaces $l$. It follows from the inverse function theorem that the complex curve $F^{-1}(l)$ is properly embedded in $\mathbb{C}^{n}$ (whether $F$ is a proper map or not), hence with respect to the induced Riemannian metric it is a complete simply connected real surface of non-positive curvature [6, p. 79].

It follows from Hadamard's theorem [3, Ch. 7, Theorem 3.1] that any two points in $F^{-1}(l)$ can be joined by a unique geodesic of $F^{-1}(l)$. Given $l \in \mathbb{P}^{n-1}$, let $w(l)$ denote the initial vector of the (unique) unit-speed geodesic along $F^{-1}(l)$ joining $p$ to $q$ and set $v(l)=d F(0) w(l) \in T_{l, 0}$. Notice that all geodesic segments are contained in a fixed compact subset of $\mathbb{C}^{n}$. The map $v$ is continuous because geodesics converge to geodesics in the $C^{2}$ 
topology (which, after passing to subsequences, is a consequence of uniform $C^{3}$ boundedness) and from the uniqueness of the geodesic along $F^{-1}(l)$. Since $v$ is nonvanishing, it is clear that $\frac{v(l)}{|v(l)|}$ defines a continuous section to the Hopf map, a contradiction in view of 2.1 ,

Remark 2.3. Notice in the previous example that if the pre-images of complex lines are not simply connected, then the geodesics along them from $p$ to $q$ may not be unique, so the construction above does not work. On the other hand, because the curvature is non-positive, there is a unique geodesic for each homotopy class of paths from $p$ to $q$, so there is at least a local finite multisection in this case: potentially pieces of these could be glued to construct a global continuous section. We conjecture that for local biholomorphisms $F: \mathbb{C}^{n} \rightarrow \mathbb{C}^{n}$ that connectedness of the pre-images alone is enough to ensure injectivity of $F$.

The proof of our main theorem is similar in spirit to Example [2.2, except that we use tangent vectors to rational curves to produce a section to the map $\pi$. The construction of the map is given below, the proof of continuity being delayed to section three.

\section{Proof of Theorem 1.1}

Taking $q=0$, suppose that $F^{-1}(0)$ contains at least two distinct points $z_{1} \neq z_{2}$. Fix the two points $w_{1}=0$ and $w_{2}=1$ in $\mathbb{C P}^{1}$ along with two nonzero real tangent vectors $0 \neq \alpha_{i} \in T_{\mathbb{C P}^{1}, w_{i}}$. Let $l$ be a complex line through 0 . We claim that

(i) There are exactly two holomorphic embeddings $T_{l}=T_{l}^{1}, T_{l}^{2}: F^{-1}(l) \rightarrow \mathbb{C P}^{1}$ such that $T_{l}\left(z_{i}\right)=w_{i}(i=1,2)$ and

$$
d F\left(z_{1}\right)\left(d T_{l}\left(z_{1}\right)\right)^{-1} \alpha_{1}=d F\left(z_{2}\right)\left(d T_{l}\left(z_{2}\right)\right)^{-1} \alpha_{2} .
$$

(ii) Letting $v_{1}$ (resp. $v_{2}$ ) be the vector in equation (11) for $T_{l}^{1}\left(\operatorname{resp} . T_{l}^{2}\right)$, we have $v_{1}=-v_{2}$.

To see this, fix a holomorphic embedding $S_{l}: F^{-1}(l) \hookrightarrow \mathbb{C P}^{1}$ that takes $z_{i}$ to $w_{i}$. By rigidity, any such embedding $F^{-1}(l) \rightarrow \mathbb{C P}^{1}$ is of the form $T_{l}=U \circ S_{l}$ for an automorphism $U: \mathbb{C P}^{1} \rightarrow \mathbb{C P}^{1}$ which fixes $w_{1}=0$ and $w_{2}=1$. Any such $U$ has the form

$$
U_{a}(z)=\frac{z}{a z+1-a}
$$

for some $a \neq 1$. Setting $b=1-a$, and using $U_{a}^{\prime}(0)=b^{-1}, U_{a}^{\prime}(1)=b$ we substitute $\left(d T_{l}\left(z_{1}\right)\right)^{-1} \alpha_{1}=b d S_{l}^{-1}(0) \alpha_{1}$ and $\left(d T_{l}\left(z_{2}\right)\right)^{-1} \alpha_{2}=b^{-1} d S_{l}^{-1}(1) \alpha_{2}$ into Equation (11) to obtain

$$
b d F\left(z_{1}\right) d S_{l}^{-1}(0) \alpha_{1}=b^{-1} d F\left(z_{2}\right) d S_{l}^{-1}(1) \alpha_{2}
$$

in the tangent space $T_{l, 0} \subset T_{\mathbb{C}^{n}, 0}$, which we identify with the subset $\mathbb{C} \cong l \subset \mathbb{C}^{n}$. Thus $b^{2} \in \mathbb{C}-\{0\}$ and we find two values for $b$, one being the negative of the other. This proves (i) and (ii) above.

In particular, both choices of $T_{l}$ yield the same real line $v(l) \subset l \subset \mathbb{C}^{n}$, hence the map $v: \mathbb{C P}^{n-1} \rightarrow \mathbb{R P}^{2 n-1}$ is a set-theoretic section of the map $\pi$ above. Using the BieberbachGronwall estimate for univalent functions on the unit disc [7, Theorem 1.3], we will show in section three that $v$ is a continuous section: this contradiction finishes the proof. 
Example 2.4. The conclusion of Theorem 1.1 may fail if the pre-image of even a single line is disconnected. Let $f: \mathbb{C} \rightarrow \mathbb{C}$ be a holomorphic function with nowhere zero derivative and which assumes the value 0 more than once. Let $F$ be the local biholomorphism of $\mathbb{C}^{2}$ defined by $F(z, w)=(f(z), w)$. If $l \subset \mathbb{C}^{2}$ is the complex line $a w_{1}+b w_{2}=0$, then $F^{-1}(l)$ is conformal to $\mathbb{C}$ if $b \neq 0$, which is a rigid domain. For $b=0$, however, the pre-image $F^{-1}(l)$ is homeomorphic to the disconnected space $f^{-1}(0) \times \mathbb{C}$.

Example 2.5. This example shows that the rationality condition in part (2) of Corollary 1.3 cannot be removed, hence the condition that $F^{-1}(l)$ be conformal to a rigid domain of $\mathbb{C P}^{1}$, rather than a compact Riemann surface of positive genus, cannot be removed from Theorem 1.1] or Corollary 1.2. Let $Y \subset \mathbb{P}^{3}$ be a general smooth surface of degree $d>2$. Then $Y$ is neither ruled nor a Steiner surface, hence has no two-dimensional families of reducible plane sections [10, Lemma II.2.4]. It follows that for a general point $p \in \mathbb{P}^{3}$, only a finite number of planes $H_{i}$ containing $p$ intersect $Y$ in a reducible curve. Let $F: Y \rightarrow \mathbb{P}^{2}$ be the projection from such a point $p$ onto a plane $\mathbb{P}^{2} \subset \mathbb{P}^{3}$. If $q \in \mathbb{P}^{2}$ and $q \notin \bigcup_{i} H_{i}$, then every line $l$ through $q$ has irreducible pre-image $F^{-1}(l)=Y \cap H$ ( $H$ spanned by $l$ and $\left.p\right)$ of arithmetic genus $p_{a}(Y \cap H)=\frac{1}{2}(d-1)(d-2)$ [8, I, Ex. 7.2(b)]. Moreover, $Y \cap H$ is smooth and irreducible for general $l$ containing $q$ by Bertini's theorem [9, Thm. 6.10], so the geometric (or topological) genus is also $p_{g}\left(F^{-1}(l)\right)=\frac{1}{2}(d-1)(d-2)$ [8, III, Remark 7.12.2].

There is an open subset $U \subset Y-\bigcup_{i} H_{i}$ such that the restriction map $\left.F\right|_{U}: U \rightarrow \mathbb{P}^{2}$ is étale 13. I, Prop. 3.8]. Removing a line $L \subset \mathbb{P}^{2}$ gives an étale morphism $F: X=U-\pi_{p}^{-1}(L) \rightarrow \mathbb{C}^{2}$ of degree $d$ such that the pre-image of every line meeting $F(X)$ is an irreducible curve. For the general such line $l$, the pre-image $F^{-1}(l)$ is a Zariski open subset of a smooth projective plane curve $\left(Y \cap H\right.$ in the discussion above) of geometric genus $\frac{1}{2}(d-1)(d-2)>0$.

Example 2.6. Let $S \subset \mathbb{R}^{3}$ be the cubic surface $x^{2}+y^{2}=z^{2}(1-z)$ obtained by rotating a nodal cubic curve about the $z$-axis and consider the projection $F: S \rightarrow \mathbb{R}^{2}$ to the $x y$-plane. The Jacobian determinant vanishes along the set $W$ consisting of the origin and the circle $z=\frac{2}{3}$, so the restricted map $X:=S-W \rightarrow \mathbb{R}^{2}$ becomes a local diffeomorphism. The map is not globally injective (points on the disk $D=\left\{(x, y): x^{2}+y^{2} \leq \frac{4}{27}\right\}$ have three pre-images) and the pre-image of a line $l$ is connected if and only if $l$ misses the disk $D$ (compare [15. Example 3.3]).

Now we complexify the above example. Take $S \subset \mathbb{C}^{3}$ to be the complex surface of the same equation and remove the locus $W$ consisting of the origin and the complex curve $z=\frac{2}{3}$ to obtain $X=S-W$. Projection to the $x y$-plane now gives a non-injective local biholomorphism $F: X \rightarrow \mathbb{C}^{2}$. Consider the complex line $l: y=a x$ through the origin: the equations of the pre-image under $F$ are $y=a x$ and $\left(1+a^{2}\right) x^{2}=z^{2}(1-z)$. If $a \neq \pm i$, then the pre-image $F^{-1}(l)$ is a connected rational curve (a parametrization is given by $\left.x=\frac{t^{2}-1-a^{2}}{t^{3}}, y=a x, z=t x\right)$, but for $a= \pm i$ the pre-image is not connected, consisting of two disjoint lines $z=0$ and $z=1$. The pre-images of the lines through the point $(0,1)$ are all connected (being zero sets of irreducible polynomials), but most of them are the complement of a smooth elliptic cubic curve by finitely many points.

Example 2.7. Theorem 1.1 fails if we remove the rigidity condition. Consider the map $F: D \times D \rightarrow \mathbb{C}^{2}$ given by $F(z, w)=\left((w+2) e^{7 z}, w\right)$, where $D \subset \mathbb{C}$ is the open unit disc. 
Then $F$ is a local biholomorphism since $\operatorname{det} J F=7(w+2) e^{7 z} \neq 0$ on $D \times D$. Now let $\mathcal{U} \subset D \times D$ be the union of all connected components through $(0,0)$ of all pre-images of complex lines $l$ passing through $(2,0)$. It follows from the inverse function theorem that the interior $\mathcal{U}^{0}$ of $\mathcal{U}$ is closed in $\mathcal{U}$. Since $(0,0) \in \mathcal{U}^{0}$ and $\mathcal{U}$ is connected, one has that $\mathcal{U}=\mathcal{U}^{0}$ is open. The restriction $\left.F\right|_{\mathcal{U}}$ is not injective, since $F(0,0)=F\left(\frac{2 \pi i}{7}, 0\right)=(2,0)$. The line $w=0$ pulls back to $D \times\{0\}$, a non-rigid domain in $\mathbb{C P}^{1}$. Restricting $F$ to a sufficiently small neighborhood $\mathcal{U}^{\prime} \subset \mathcal{U}$ of $D \times\{0\}$, it is clear that the pull-back of every line $l$ through $(2,0)$ is a connected set which is conformal to an open non-rigid subset of $\mathbb{C P}^{1}$.

\section{The CONTINUity PROOF}

In this section we complete the proof of Theorem 1.1 by showing continuity of the map $v: \mathbb{C P}^{n-1} \rightarrow \mathbb{R P}^{2 n-1}$ there, constructed as follows. Starting with a local biholomorphism $F: X \rightarrow \mathbb{C}^{n}$ such that $z_{1} \neq z_{2}$ and $F\left(z_{1}\right)=F\left(z_{2}\right)=0$, we assumed that for each complex line $l \in \mathbb{C P}^{n-1}$ through the origin, $F^{-1}(l)$ is a rigid domain in $\mathbb{C P}^{1}$, hence a connected rational curve. Fixing $w_{1}=0$ and $w_{2}=1$ in $\mathbb{C P}^{1}$ and real tangent vectors $\alpha_{i} \in T_{\mathbb{P}^{1}, w_{i}}$, we showed that there are exactly two embeddings $T_{l}^{1}, T_{l}^{2}: F^{-1}(l) \rightarrow \mathbb{C P}^{1}$ sending $z_{i}$ to $w_{i}(i=1,2)$ and satisfying

$$
d F\left(z_{1}\right)\left(d T_{l}^{j}\left(z_{1}\right)\right)^{-1} \alpha_{1}=d F\left(z_{2}\right)\left(d T_{l}^{j}\left(z_{2}\right)\right)^{-1} \alpha_{2} \in \mathbb{C}^{n}
$$

for $j=1,2$. Furthermore, both embeddings give rise to the same real line through 0 (we now drop the superscript and write $T_{l}$ for either of the two embeddings). In particular, we have defined a map $v: \mathbb{C P}^{n-1} \rightarrow \mathbb{R} \mathbb{P}^{2 n-1}$. such that $v(l)=p(\kappa(l))$, where $p: \mathbb{C}^{n}-\{0\} \rightarrow \mathbb{R} \mathbb{P}^{2 n-1}$ is the natural projection and $\kappa: \mathbb{C P}^{n-1} \rightarrow \mathbb{C}^{n}-\{0\}$ is given by $\kappa(l)=d F\left(z_{1}\right)\left(d T_{l}\left(z_{1}\right)\right)^{-1} \alpha_{1}$. We will now show that $v$ is continuous; in other words, for any convergent sequence $l_{q} \rightarrow l$ in $\mathbb{C P}^{n-1}$, we will show that $v\left(l_{q}\right) \rightarrow v(l)$.

The salient feature is that the submanifolds $F^{-1}\left(l_{q}\right)$ converge to $F^{-1}(l)$ uniformly over compact subsets of $X$. Since we are proving that $v\left(l_{q}\right) \rightarrow v(l)$ for any sequence $l_{q} \rightarrow l$, it suffices to show that $v\left(l_{q}\right)$ has a subsequence converging to $v(l)$. Indeed, this shows that $v(l)$ is the only accumulation point. We will achieve this by essentially (though not literally) writing the map $T_{l}: F^{-1}(l) \rightarrow \mathbb{C P}^{1}$ as a limit of the maps $T_{q}=T_{l_{q}}$. To this end, let $S_{q}$ be a sequence in the unitary group $U(n)$ such that $S_{q} \rightarrow I_{n}$ and $S_{q}(l)=l_{q}$.

Fix a complete Riemannian metric on $X$. Since $F^{-1}(l)$ is connected, there is a closed geodesic ball $K_{0}=\overline{B_{R}\left(z_{1}\right)}$ of some radius $R>0$ centered at $z_{1}$ such that $z_{1}, z_{2}$ lie in the same connected component $C_{0}$ of the compact domain $K_{0} \cap F^{-1}(l)$ for the Riemann surface $F^{-1}(l)$. Setting $K_{m}=\overline{B_{R+m}\left(z_{1}\right)}$, we obtain a countable increasing exhaustion $\left\{K_{m}\right\}_{m \geq 1}$ of $X$. Clearly $z_{1}, z_{2}$ lie in the same connected component $C_{m}$ of $K_{m} \cap F^{-1}(l)$ for each $m \geq 1$. Since $K_{m}$ is compact, there is $\delta=\delta(m)>0$ such that

(1) $\left.F\right|_{B_{\delta}(z)}$ is a biholomorphism onto its image for all $z \in K_{m}$.

(2) $\left.F\right|_{B_{\delta}(z) \cup B_{\delta}\left(z^{\prime}\right)}$ is injective whenever $B_{\delta}(z) \cap B_{\delta}\left(z^{\prime}\right)$ is nonempty.

Indeed, for $x \in K_{m}$, there exists $\delta_{x}>0$ such that $\left.F\right|_{B_{\delta_{x}}(x)}$ is a biholomorphism onto its image. The open cover $\left\{B_{\frac{\delta_{x}}{4}}(x)\right\}_{x \in K_{m}}$ for $K_{m}$ has a finite subcover $\left\{B_{\frac{\delta_{k}}{4}}\left(x_{k}\right)\right\}_{k=1}^{r}$ and we set $\delta=\delta(m)=\min _{1 \leq k \leq r}\left\{\frac{\delta_{k}}{4}\right\}$. For $z \in B_{\delta}\left(x_{k}\right) \cap K_{m}$, we have $B_{\delta}(z) \subset B_{2 \delta}\left(x_{k}\right)$ which implies 
condition 1 since $F$ takes the larger ball biholomorphically onto its image. If $B_{\delta}\left(z^{\prime}\right)$ meets $B_{\delta}(z)$, then $B_{\delta}\left(z^{\prime}\right) \subset B_{4 \delta}\left(x_{k}\right)$ so that $B_{\delta}(z) \cup B_{\delta}\left(z^{\prime}\right) \subset B_{4 \delta}\left(x_{k}\right)$ and the restriction of $F$ to the latter ball is injective, which checks condition (2). Setting $\delta_{m}=\min _{k \leq m} \delta(k)$ we obtain a nonincreasing sequence with the same properties.

Now fix $m \geq 1$. The set

$$
S=\left\{(a, z) \in X \times\left(F^{-1}(l) \cap K_{m}\right): d(a, z)=\frac{\delta_{m}}{2}\right\}
$$

is compact, hence the continuous function $G(a, z)=|F(a)-F(z)|$ achieves its minimum value $\lambda$ on $S$; moreover $\lambda>0$ because $\left.F\right|_{B_{\delta_{m}}(z)}$ is injective for each $z \in F^{-1}(l) \cap K_{m}$. For $\mu=\frac{\lambda}{2}$ we have $B_{\mu}(F(z)) \subset F\left(B_{\delta_{m}}(z)\right)$ for all $z \in F^{-1}(l) \cap K_{m}$. The convergence $S_{q} \rightarrow I$ yields $N_{m}>0$ such that the absolute values of the entries of the matrix $S_{q}-I_{n}$ are all less than $\frac{\mu}{n^{2} M}$ for $q \geq N_{m}$, where

$$
M=M(m)=\sup _{z \in F^{-1}(l) \cap K_{m}}|F(z)| .
$$

With these choices, we have that $\left|S_{q}(F(z))-F(z)\right|<\mu$ for $z \in F^{-1}(l) \cap K_{m}$ and $q \geq N_{m}$ so that $S_{q}(F(z)) \in F\left(B_{\delta_{m}}(z)\right)$. As above, we may assume that the $N_{m}$ are nondecreasing.

For $\delta_{m}$ and $N_{m}$ as above, we have thus constructed well-defined holomorphic injections $\phi_{m, q}: C_{m} \hookrightarrow F^{-1}\left(l_{q}\right)$ for $q \geq N_{m}$ given by

$$
\phi_{m, q}(z)=\left[\left(\left.F\right|_{B_{\delta_{m}(z)}}\right)^{-1} \circ S_{q} \circ F\right](z) .
$$

By construction it is clear that $\phi_{m, q}$ fixes $z_{1}, z_{2}$ and that $\left.\phi_{m^{\prime}, q}\right|_{C_{m}}=\phi_{m, q}$ for $m^{\prime} \geq m$ and $q \geq N_{m}$. Injectivity follows from condition $(2)$ on $\delta(m)$ above. Composition with $T_{q}$ yields injective holomorphic maps

$$
\psi_{m, q}=T_{q} \circ \phi_{m, q}: C_{m} \hookrightarrow \mathbb{C P}^{1}
$$

for $q \geq N_{m}$ which satisfy $\psi_{m, q}\left(z_{i}\right)=w_{i}$ and $\left.\psi_{m^{\prime}, q}\right|_{C_{m}}=\psi_{m, q}$ for $m^{\prime} \geq m$. Note that we will make a convenient choice of identification $\mathbb{C P}^{1}=\mathbb{C} \cup\{\infty\}$ later in the proof.

We will need the following compactness result in the proof of Lemma 3.2 below:

Lemma 3.1. Let $D \subset \mathbb{C}$ be the open unit disc, $0 \neq a \in D$ and $0 \neq b \in \mathbb{C}$. Then

$$
\mathcal{F}_{a, b}=\{\text { holomorphic injections } f: D \hookrightarrow \mathbb{C} \mid f(0)=0 \text { and } f(a)=b\}
$$

is a normal family of functions.

Proof. Any univalent holomorphic function $f$ on $D$ that vanishes at 0 satisfies the well-known estimate of Bieberbach and Gronwall (see, for example [7, Theorem 1.3] for the normalized estimate)

$$
\frac{\left|f^{\prime}(0)\right| r}{(1+r)^{2}} \leq|f(z)| \leq \frac{\left|f^{\prime}(0)\right| r}{(1-r)^{2}}
$$

where $r=|z|$. Evaluating the inequality on the left at $z=a$ gives an upper bound on $\left|f^{\prime}(0)\right|$. The inequality on the right now shows that $|f(z)|$ is uniformly bounded over compact subsets of $D$, with a bound independent of $f$. By Montel's theorem $\mathcal{F}_{a, b}$ is normal, that is $\overline{\mathcal{F}_{a, b}}$ is compact in the space of holomorphic functions on $D$, endowed with the topology of uniform convergence over compact subsets. 
Lemma 3.2. Fix $m \geq 1$ and an identification $\mathbb{C P}^{1}=\mathbb{C} \cup\{\infty\}$. Let $\left\{\varphi_{k}\right\}$ be a subsequence of $\left\{\psi_{m, q}\right\}_{q \geq N_{m}}$ such that each $\varphi_{k}$ has a pole $p_{k}$ and $\left\{p_{k}\right\}$ converges to a point $x$ in the interior of $C_{m}$. Then $\left\{\varphi_{k}\right\}$ has a further subsequence converging uniformly over compact subsets of the interior of $C_{m}-\{x\}$ to a holomorphic injection of the interior of $C_{m}-\{x\}$ into $\mathbb{C}=\mathbb{C P}^{1}-\{\infty\}$.

Proof. To set notation, write $\varphi_{k}=\psi_{m, s_{0}(k)}$ for some strictly increasing function $s_{0}: \mathbb{N} \rightarrow \mathbb{N}$ with $s_{0}(1) \geq N_{m}$. Cover $C_{m}-\{x\}$ with countably many embedded holomorphic disks $\xi_{r}: D \hookrightarrow C_{m}-\{x\}$ in such a way that $\xi_{r}(0)=z_{1}, z_{2} \in \xi_{r}(D)$ but $x \notin \overline{\xi_{r}(D)}$.

Consider the $r$ th embedded holomorphic disk $\xi_{r}: D \hookrightarrow C_{m}-\{x\}$. Then $p_{k} \notin \xi_{r}(D)$ for $k$ sufficiently large because $x \notin \overline{\xi_{r}(D)}$, hence the maps $\varphi_{k} \circ \xi_{r}$ belong to class $\mathcal{F}_{\xi_{r}^{-1}\left(z_{2}\right), w_{2}}$ of Lemma 3.1 (their images lie in $\mathbb{C}$ via the identification $\mathbb{C P}^{1}=\mathbb{C} \cup\{\infty\}$ ). In particular, there exists a subsequence $\varphi_{s_{1}(k)} \circ \xi_{r}$ converging uniformly over the compact sets of $D$ by normality of $\mathcal{F}_{\xi_{r}^{-1}\left(z_{2}\right), w_{2}}$. Composing with $\xi_{r}^{-1}$, the subsequence $\varphi_{s_{1}(k)}$ converges uniformly over the compact subsets of the topological disc $\xi_{r}(D) \subset C_{m}-\{x\}$ to a holomorphic map taking values in $\mathbb{C}=\mathbb{C P}^{1}-\{\infty\}$. Since $\varphi_{s_{1}(k)}\left(z_{i}\right)=w_{i}(i=1,2)$, we see that the nonconstant limit function is the local uniform limit of a sequence of injective holomorphic functions. By a well-known theorem of Hurwitz, the limit function is itself injective.

Taking $r=1$ above, $\varphi_{k}$ has a subsequence $\varphi_{s_{1}(k)}$ which converges uniformly over compact sets in $\xi_{1}(D)\left(s_{1}: \mathbb{N} \rightarrow \mathbb{N}\right.$ strictly increasing as above). Applying the previous paragraph with $r=2$ and the subsequence $\varphi_{s_{1}(k)}$, we obtain a further subsequence $\varphi_{s_{2}(k)}$ with the same property with respect to both $\xi_{1}(D)$ and $\xi_{2}(D)$. Continuing in this fashion, we obtain a countable sequence of subsequences $\varphi_{s_{r}(k)}$ (each uniformly convergent on compact subsets of $\xi_{p}(D)$ for $\left.1 \leq p \leq r\right)$, each being extracted from the previous: $s_{1}(\mathbb{N}) \supset s_{2}(\mathbb{N}) \supset s_{3}(\mathbb{N}) \ldots$ Thus the diagonal subsequence $\varphi_{s_{r}(r)}$ converges uniformly to an injective holomorphic function on every compact subset of $C_{m}-\{x\}$. This proves Lemma 3.2.

Taking $m=1$, we now make a choice of both $\infty \in \mathbb{C P}^{1}$ and a subsequence $\varphi_{k}$ of $\psi_{1, q}$ for which the hypotheses of Lemma 3.2 holds. Start with any point $a \in \mathbb{C P}^{1}$. If there happens to be a subsequence $\psi_{1, q_{k}}$ and $p_{k} \in C_{1}$ with $\psi_{1, q_{k}}\left(p_{k}\right)=a$ and $p_{k} \rightarrow x$ with $x$ in the interior of $C_{1}$, then we simply take $\infty=a$ and $\varphi_{k}=\psi_{1, q_{k}}$. The alternatives are that $(1) a \in \psi_{1, q}\left(C_{1}\right)$ for only finitely many $q$ or $(2) a \in \psi_{1, q}\left(C_{1}\right)$ for infinitely many $q$, but for all subsequences $p_{k}, q_{k}$ with $\psi_{1, q_{k}}\left(p_{k}\right)=a$ and $p_{k} \rightarrow x$, we have $x \in \partial C_{1}$. In either case, we can find a subsequence $q_{k}$ and an embedded holomorphic disk $\xi: D \hookrightarrow C_{1}$ such that $\xi(0)=z_{1}, z_{2} \in \xi(D)$ and $a \notin \psi_{1, q_{k}}(\xi(D))$ for $k$ sufficiently large. Thus $\psi_{1, q_{k}} \circ \xi: D \hookrightarrow \mathbb{C}=\mathbb{C P}^{1}-\{a\}$ for $k>>0$ and by Lemma 3.1 we can find a further subsequence $\psi_{1, q_{k_{l}}} \circ \xi$ converging uniformly over compact subsets. Now fix $y \in D$, set $x=\xi(y)$ and take $\infty=\lim _{l \rightarrow \infty} \psi_{1, q_{k_{l}}}(x)$. Thus we obtain a subsequence $\varphi_{k}=\psi_{1, q_{k_{l}}}$ of $\psi_{1, q}$ for which every further subsequence forcibly has poles which converge to $x$ in the interior of $C_{1}$ and we may apply Lemma 3.2 .

Finally we vary $m \geq 1$. Starting with $m=1$, choose $\infty \in \mathbb{C P}^{1}$ and $\varphi_{k}$ as above and apply Lemma 3.2 to obtain a subsequence $\varphi_{s_{1}(k)}$ which converges uniformly to an injective holomorphic function on compact subsets of the interior of $C_{1}-\{x\}$. Setting $B=\min \left\{k: s_{1}(k) \geq N_{2}-1\right\}$ and $t_{1}(k)=s_{1}(k+B)$ we have a subsequence $\varphi_{t_{1}(k)}$ of $\psi_{2, n}$ whose restrictions to $C_{1}$ form a subsequence of $\varphi_{s_{1}(k)}$; moveover, the hypotheses of Lemma 3.2 
hold automatically by the compatibility condition on the maps $\psi_{m, q}$, that is, $\left.\psi_{m^{\prime}, q}\right|_{C_{m}}=\psi_{m, q}$ for $m^{\prime} \geq m$. Thus applying Lemma 3.2 with $m=2$ gives a further subsequence that converges uniformly to an injective holomorphic function on compact subsets of the interior of $C_{2}-\{x\}$. Continuing in this fashion, we obtain a countable sequence $\left\{\varphi_{s_{m}(k)}\right\}_{m \geq 1}$ of subsequences, each being extracted from the restriction of the next: $\varphi_{s_{m}(k)}$ converges uniformly over compact subsets to an injective holomorphic function on the interior of $C_{m}-\{x\}$. Taking the diagonal subsequence $\varphi_{s_{m}(m)}$ yields

Lemma 3.3. There is an injective holomorphic map $\eta: F^{-1}(l)-\{x\} \hookrightarrow \mathbb{C}=\mathbb{C P}^{1}-\{\infty\}$ such that for each fixed $m \geq 1$, the restriction of $\eta$ to the interior of $C_{m}-\{x\}$ is the local uniform limit of a subsequence $\psi_{m, q_{l}}$ of $\psi_{m, q}$.

The map $\eta$ extends to a holomorphic injection $\tilde{\eta}: F^{-1}(l) \rightarrow \mathbb{C P}^{1}$ such that $\tilde{\eta}\left(z_{i}\right)=w_{i}$ for $i=1,2$. For $z$ in the interior of $\in C_{1}-\{x\}$ (say $z$ near $z_{1}$ or $z_{2}$ ), we use Lemma 3.3 to write

$$
\tilde{\eta}(z)=\lim _{k \rightarrow \infty} \psi_{1, q_{k}}(z)
$$

as a local uniform limit for some subsequence $q_{k}$. Since the sequence of derivatives also converges, we have

$$
d F\left(z_{1}\right) \circ\left(d \tilde{\eta}\left(z_{1}\right)\right)^{-1}\left(\alpha_{1}\right)=\lim _{k \rightarrow \infty} d F\left(z_{1}\right) \circ\left(d \psi_{1, q_{k}}\left(z_{1}\right)\right)^{-1}\left(\alpha_{1}\right) .
$$

Since $\psi_{1, q}=T_{q} \circ \phi_{1, q}$ and $\phi_{1, q}(z)=\left[\left(\left.F\right|_{\left(B_{\delta_{1}(z)}\right)}\right)^{-1} \circ S_{q} \circ F\right](z)$, this becomes

$$
\lim _{k \rightarrow \infty}\left[\left(S_{q_{k}}\right)^{-1} \circ d F\left(z_{1}\right) \circ\left(d T_{q_{k}}\left(z_{1}\right)\right)^{-1}\left(\alpha_{1}\right)\right]
$$

but $\lim _{k \rightarrow \infty} S_{q_{k}}=I=n \times n$ identity matrix, so we conclude that

$$
d F\left(z_{1}\right) \circ\left(d \tilde{\eta}\left(z_{1}\right)\right)^{-1}\left(\alpha_{1}\right)=\lim _{k \rightarrow \infty} d F\left(z_{1}\right) \circ\left(d T_{q_{k}}\left(z_{1}\right)\right)^{-1}\left(\alpha_{1}\right) .
$$

Analogously,

$$
d F\left(z_{2}\right) \circ\left(d \tilde{\eta}\left(z_{2}\right)\right)^{-1}\left(\alpha_{2}\right)=\lim _{k \rightarrow \infty} d F\left(z_{2}\right) \circ\left(d T_{q_{k}}\left(z_{2}\right)\right)^{-1}\left(\alpha_{2}\right) .
$$

By the definition of $T_{q_{k}}$, one has

$$
d F\left(z_{1}\right) \circ\left(d T_{q_{k}}\left(z_{1}\right)\right)^{-1}\left(\alpha_{1}\right)=d F\left(z_{2}\right) \circ\left(d T_{q_{k}}\left(z_{2}\right)\right)^{-1}\left(\alpha_{2}\right),
$$

so that

$$
d F\left(z_{1}\right) \circ\left(d \tilde{\eta}\left(z_{1}\right)\right)^{-1}\left(\alpha_{1}\right)=d F\left(z_{2}\right) \circ\left(d \tilde{\eta}\left(z_{2}\right)\right)^{-1}\left(\alpha_{2}\right) .
$$

Together with $\tilde{\eta}\left(z_{i}\right)=w_{i}(i=1,2)$, the last relation shows that $\tilde{\eta}$ is equal to one of the two maps $T_{l}^{j}$. Taking images in $\mathbb{R P}^{2 n-1}$ one now sees that $v\left(l_{q_{k}}\right) \rightarrow v(l)$. This shows that the section $v: \mathbb{C P}^{n-1} \rightarrow \mathbb{R} \mathbb{P}^{2 n-1}$ of the natural map $\pi: \mathbb{R P}^{2 n-1} \rightarrow \mathbb{C P}^{n-1}$ is continuous. Following the earlier part of the proof, this contradiction proves Theorem 1.1 .

\section{REFERENCES}

[1] H. Bass, E. Connell and D. Wright, The Jacobian Conjecture: Reduction of the degree and formal expansion of the inverse, Bull. Amer. Math. Soc. 7 (1982) 287-330.

[2] L. de Branges, A proof of the Bieberbach Conjecture, Acta Math. 154 (1985) 137-152.

[3] M. do Carmo, Riemannian Geometry, Birkhäuser Boston, Boston, 1992.

[4] A. V. den Essen, Polynomial Automorphisms and the Jacobian Conjecture, Progress in Mathematics 190, Birkhäuser Verlag, Basel, 2000. 
[5] S. Gong, The Bieberbach Conjecture (translated from the 1989 Chinese original and revised by the author, with preface by C. H. Fitzgerald), AMS/IP Studies in Advanced Mathematics 12, American Mathematical Society, Providence, RI, International Press, Cambridge, MA, 1999.

[6] P. Griffiths and J. Harris, Principles of algebraic geometry, Pure and Applied Mathematics, WileyInterscience [John Wiley \& Sons], New York, 1978.

[7] W. Hayman, Multivalent functions (second edition), Cambridge Tracts in Mathematics and Mathematical Physics 110, Cambridge University Press, Cambridge 1994.

[8] R. Hartshorne, Algebraic Geometry, Graduate Texts in Mathematics 52, Springer-Verlag, New YorkHeidelberg, 1977.

[9] J. P. Jouanolou, Théorèmes de Bertini et Applications Progress in Mathematics 42, Birkhäuser, Boston, 1983.

[10] A. F. Lopez, Noether-Lefschetz theory and Picard group of projective surfaces, Memoirs of the AMS 438 (1991).

[11] J. Kollár, Rational Curves on Algebraic Varieties, Ergebnisse der Mathematik und ihrer Grenzgebiete 32, Springer-Verlag, Berlin Heidelberg New York, 1996.

[12] G. Meisters, Inverting polynomial maps of n-space by solving differential equations, in "Delay and Differential Equations, Proceedings in Honor of George Seifert, Ames, Iowa, Oct. 18-19, 1991", 107-166, World Sci. Publishing, River Edge, NJ, 1992.

[13] J. S. Milne, Étale cohomology, Princeton Mathematical Series 33, Princeton University Press, Princeton, N.J., 1980.

[14] S. Mori, Projective manifolds with ample tangent bundles, Ann. of Math. (2) 110 (1979) 593-606.

[15] S. Nollet and F. Xavier, Global inversion via the Palais-Smale condition, Disc. Cont. Dynam. Sys. - Ser. A 8 (2002) 17-28.

[16] T. Parthasarathy, On global univalence theorems, Lecture Notes in Mathematics 977, Springer-Verlag, Berlin-Heidelberg-New York 1983.

[17] Ch. Pommerenke, Univalent functions (with a chapter on quadratic differentials by Gerd Jensen), Studia Mathematica/Mathematische Lehrbücher XXV, Vandenhoeck \& Ruprecht, Göttingen, 1975.

[18] J. P. Serre, Géométrie algébrique et géométrie analytique, Ann. Inst. Fourier, Grenoble 6 (1955) 1-42.

[19] B. Smyth and F. Xavier, Injectivity of local diffeomorphisms from nearly spectral conditions, J. Diff. Equs. 130 (1996) 406-414.

[20] F. Xavier, Injectivity as a transversality phenomenon in geometries of negative curvature, Ill. Jour. Math. 43 (1999) 256-263.

Department of Mathematics, Texas Christian University, Fort Worth, TX 76129

E-mail address: s.nollet@tcu.edu

Department of Mathematics, University of Notre Dame, Notre Dame, IN 46556

E-mail address: fxavier@nd.edu 